\title{
POPULATION STUDY ARTICLE Technoference: longitudinal associations between parent technology use, parenting stress, and child behavior problems
}

\author{
Brandon T. McDaniel ${ }^{1}$ and Jenny S. Radesky ${ }^{2}$
}

\begin{abstract}
BACKGROUND AND OBJECTIVES: Heavy parent digital technology use has been associated with suboptimal parent-child interactions and internalizing/externalizing child behavior, but directionality of associations is unclear. This study aims to investigate longitudinal bidirectional associations between parent technology use and child behavior, and understand whether this is mediated by parenting stress.

METHODS: Participants included 183 couples with a young child (age $0-5$ years, mean $=3.0$ years) who completed surveys at baseline, 1, 3 and 6 months. Cross-lagged structural equation models of parent technology interference during parent-child activities, parenting stress, and child externalizing and internalizing behavior were tested.

RESULTS: Controlling for potential confounders, we found that across all time points (1) greater child externalizing behavior predicted greater technology interference, via greater parenting stress; and (2) technology interference often predicted greater externalizing behavior. Although associations between child internalizing behavior and technology interference were relatively weaker, bidirectional associations were more consistent for child withdrawal behaviors.

CONCLUSIONS: Our results suggest bidirectional dynamics in which (a) parents, stressed by their child's difficult behavior, may then withdraw from parent-child interactions with technology and (b) this higher technology use during parent-child interactions may influence externalizing and withdrawal behaviors over time.
\end{abstract}

Pediatric Research (2018) 84:210-218; https://doi.org/10.1038/s41390-018-0052-6

\section{INTRODUCTION}

Despite being a cultural norm, ${ }^{1}$ mobile phone use in public and private spaces is frequently described as an uncomfortable interruption by users ${ }^{2}$ and their social companions. ${ }^{3}$ Studies suggest that adults experience more frustration and conflict, ${ }^{4,5}$ less in-depth conversation, ${ }^{6}$ and lower sense of empathy ${ }^{7}$ when mobile devices are used during social interactions. Parents are now estimated to use digital media (e.g., television, computers, smartphones, tablets) an average of $9 \mathrm{~h}$ per day, ${ }^{8}$ with $\sim 3 \mathrm{~h}$ per day on their smartphones. ${ }^{9}$

Parent mobile device use has received particular scrutiny because the portability of devices has facilitated their use during many family activities such as meals, playtime, and bedtime, ${ }^{10}$ which have an important role in shaping child social-emotional health. ${ }^{11-13}$ In naturalistic observations of families, Radesky and colleagues recorded less conversation and more parent hostility in response to child bids for attention when parents' attention was absorbed in their mobile devices. ${ }^{14}$ Subsequent studies have documented associations between parent mobile device use and fewer parent-child interactions during videotaped eating tasks, ${ }^{15}$ as well as lower responsiveness to child bids for attention on the playground. ${ }^{16}$ When interviewed about the experience of parenting in the presence of mobile devices, parents reported feeling emotionally and cognitively affected by their mobile device use in ways that can make it difficult to read and respond to child behavioral cues. $^{2}$

However, only one prior study has examined links between parent device use and child behavioral outcomes. McDaniel and
Radesky found cross-sectional associations between parent technology interference ("technoference," defined by McDaniel and Coyne ${ }^{4}$ as everyday interruptions in interpersonal interactions or time spent together that occur due to digital and mobile technology devices) and higher child externalizing (e.g., tantrums, emotional reactivity) and internalizing (e.g., anxiety, withdrawal) behavior problems-especially for mother-child activity interruptions. ${ }^{17}$ However, directionality of these associations remains unclear. While it is plausible that interrupted parent-child play or reduced parent responsiveness could contribute to child behavioral problems, it is also possible that parents use technology to cope with parenting stresses-e.g., to escape from parenting demands $s^{3}$ or to connect with other parents for social support. ${ }^{18}$ In semi-structured interviews, parents have described using mobile devices when needing a break from difficult child behavior, relieving stress through use of entertainment apps, or to keep their household quiet. ${ }^{2}$

Another limitation of the existing literature is the lack of longitudinal studies; thus, examination of transactional processes $^{19}$ (i.e., the bidirectional influences of child and environment over time) in parent media use and child outcomes has not been possible. The aim of the present study was to build upon prior cross-sectional findings by examining transactional associations between technoference in parent-child activities and child behavior in a cohort of mothers, fathers, and their young children. In line with prior work, ${ }^{17}$ we hypothesized that more frequent technoference in daily mother-child and father-child interactions would predict higher ratings of child internalizing and

${ }^{1}$ Illinois State University, Normal, IL, USA and ${ }^{2}$ University of Michigan Medical School, Ann Arbor, MI, USA

Correspondence: Brandon T. McDaniel (btmcdaniel.phd@gmail.com)

Received: 19 October 2017 Revised: 23 February 2018 Accepted: 25 March 2018

Published online: 13 June 2018 
externalizing behavior $(\mathrm{H} 1)$; higher ratings of child internalizing and externalizing behavior would predict higher parenting stress $(\mathrm{H} 2)$; and higher parenting stress would predict more frequent technology interference (as parents withdraw to technology to escape or self-regulate, $\mathrm{H} 3$ ).

\section{PATIENTS AND METHODS}

Participants \& procedure

Participants included mothers and fathers from 183 couples with a young child who took part in the Daily Family Life Project, ${ }^{20}$ a longitudinal study of parenting and family relationships conducted from 2014 to 2016. Participants were recruited through letters and phone calls to families who were part of a family research database in a Northeastern U.S. state, as well as via flyers in the local community. Announcements were also posted to various online resources and listservs in order to expand our reach to individuals in other areas of the U.S. To be eligible to participate, individuals had to be at least 18 years old, a parent of a child age 5 years or younger, speak English, and currently live with their child and spouse/partner. Their spouse/partner also had to be willing to participate. Participants were emailed a survey link through which they completed informed consent and a baseline online survey via Qualtrics. Participants completed follow-up online assessments at $\sim 1,3$, and 6 months.

In the present study, we first excluded 11 families whose child was younger than 1 year at baseline, since the behavior rating items were not standardized for infants. We utilized data from all remaining parents who had data from at least one time point, which resulted in an analytic sample of 337 parents (171 mothers and 166 fathers; $92 \%$ of the original sample of 366 parents); $70 \%$ of these 337 parents had data across all time points. In the analytic sample of 337 parents, families resided in the following U.S. regions: $54 \%$ Northeast, $16 \%$ Midwest, $15 \%$ South, and $15 \%$ West. On average, mothers were 31.7 years old $(S D=4.3$; range $22-42)$, and fathers were $33.3(S D=4.9$; range $22-52)$. Most families (61\%) had more than one child $(M=1.90, S D=0.91)$, and the index child was 3.0 years old on average $(S D=1.2 ;$ Range $=1.0-5.5$ years; $55 \%$ female). Most parents were married (94\%), and had at least a Bachelor's degree (72\%). The race/ethnicity breakdown was $91 \%$ Caucasian, 3\% Latino, 2\% Black/African American, 2\% Asian American, and $2 \%$ Other. Median yearly household income was $\sim 69,500 \quad(M=\$ 74,870, S D=\$ 39,470)$, with $21 \%$ of families reporting some form of state or federal assistance (e.g., medical assistance, food stamps). Utilizing chi-squares and $t$-tests, we found that parents in our analytic sample were in a longer relationship $(t(360)=1.945, p=0.052)$ and had more children $(t$ $(360)=3.79, p<0.001)$ than excluded participants; the samples were otherwise similar.

\section{Measures}

Technoference in parent-child activities. At each data collection wave, technoference (i.e., technology interference) in mother-child activities and father-child activities was assessed via mother and father self-report. Items were adapted from the Technology Device Interference Scale (TDIS), ${ }^{4}$ a measure of technoference in couple relationships that is associated with couple relationship health. ${ }^{4}$ Instead of assessing duration of parent media use, this scale measures the extent to which different forms of technology intrude in or interrupt interpersonal interactions and activities during daily routines; the scale used in the current study was reworded to refer specifically to interactions with one's child, and has been used in in prior research. ${ }^{17}$ Items asked, "On a typical day, about how many times do the following devices interrupt a conversation or activity you are engaged in with your child?" The 6 items on the scale included: ${ }^{1}$ cellphone/smartphone, ${ }^{2}$ television, ${ }^{3}$ computer, $^{4}$ tablet, ${ }^{5}$ iPod, and ${ }^{6}$ video game console. Parents responded to each item on a 7-point scale ranging from 0 (none) to 6 (more than 20 times). As this is a count measure and we expected there to be variability (as opposed to consistency) within individuals' responses, it was not appropriate to calculate Cronbach's alpha ${ }^{17}$ (however, the alpha ranged from 0.69 to 0.82 across time points). Parents were queried about different devices separately because of the assumption that different modes of technology use may interfere with parent-child activities to varying degrees, yet the level of technoference from various devices was often correlated (inter-item correlations 0.24 to 0.71 , $p s<0.001$ ). Items were therefore averaged, with higher scores representing more frequent technoference in parent-child activities.

Child externalizing and internalizing behavior problems. At each data collection wave, parents completed the internalizing (36 items) and externalizing scales (24 items) of the Child Behavioral Checklist (CBCL). ${ }^{21}$ These items ask parents to rate their child now or within the past 2 months on a 3-point scale ranging from 0 (not true) to 2 (very true or often true). Internalizing consists of items such as "whining," "sulks a lot," and "feelings are easily hurt." Externalizing consists of items such as "can't sit still, restless, or hyperactive," "easily frustrated," and "temper tantrums or hot temper." Items were summed to produce separate mother and father ratings of internalizing and externalizing child behavior (Cronbach's alphas for internalizing ranged from 0.89 to 0.94 across time points; alphas for externalizing ranged from 0.92 to 0.93 ). We then converted raw sum scores to normed externalizing and internalizing $T$-scores for analysis. Additionally, we conducted posthoc analyses with the internalizing subscales (Emotional Reactivity, Anxiety/Depression, Somatic Complaints, and Withdrawal) and externalizing subscales (Attention Problems and Aggression).

Parenting Stress. At each data collection wave, parents completed 27 items from the Parenting Stress Index (PSI). ${ }^{22}$ We used 27 items from the 36-item PSI Short Form due to lower factor loadings on 9 of the items, as others have done. ${ }^{23,22}$ Items were averaged to produce an overall stress score (Cronbach's alphas ranged from 0.91 to 0.94 across time points).

Potential confounding variables. At baseline, parents reported their age, educational attainment, marital status, race/ethnicity, family composition, household income, and child's age, gender, and health. They also completed measures of coparenting quality, depressive symptoms, and reported on their child's daily duration of screen media use.

As this sample consists of two-parent families, we controlled for coparenting quality-or how well parents work together in childrearing ${ }^{23}$ - which has been associated with child behavior problems $^{24}$ and technoference. ${ }^{10}$ Both parents completed the Coparenting Relationship Scale, ${ }^{23}$ a 35-item scale (e.g., "When I'm at my wits end as a parent, partner gives me extra support I need" and "My partner undermines my parenting") rated on a 7-point scale $(0=$ not true of us to $6=$ very true of us). After reverse coding negatively worded items, items were averaged to produce an overall score with higher scores indicating higher coparenting quality (Cronbach's alpha $=0.94)$.

Depressive symptoms were measured utilizing the validated Center for Epidemiologic Studies Depression Scale (CES-D). ${ }^{25}$ Participants rated how often they experienced 20 symptoms (e.g., "I felt depressed" and "I felt sad") in the past week on a 4-point scale ranging from 0 (rarely or none of the time, less than 1 day) to 3 (most or all of the time, 5 to 7 days). Items were averaged to produce an overall depression score (Cronbach's alpha $=0.89$ ). We controlled for depressive symptoms as depressed mood has been associated with quality of parent-child interactions ${ }^{26}$ and greater relationship technoference. $^{4}$

At baseline, parents rated how much time, on a typical day, their child spent using screen media devices across 8 items (e.g., computer, TV, smartphone, tablet, video games) on an 11-point 
scale ranging from 0 (None) to 10 (7 or more hours). Items were summed to produce an overall child screen use score (Cronbach's alpha $=0.78)$. We controlled for child screen media use because it is strongly associated with both parent media use ${ }^{9}$ and child social-emotional outcomes. $^{27}$

\section{Data Analysis}

We utilized structural equation modeling (SEM) to test two separate models, one for child externalizing and one for child internalizing, of: (H1) more frequent technoference in parent-child interactions predicting higher ratings of child behavior problems; $(\mathrm{H} 2)$ higher ratings of child behavior problems predicting higher parenting stress; and $(\mathrm{H} 3)$ higher parenting stress predicting more frequent technoference in parent-child activities. The models were tested utilizing AMOS. $^{28}$ Standardized estimates are shown for the models in Figs. 1 and 2. Potential confounders including parent characteristics, child age, child screen use, parent depressive symptoms, and coparenting quality were entered into the models, but were removed from the final models as results did not change significantly. Structural equation modeling was utilized as this allowed us to examine the complex cross-lagged paths between our various predictors and outcomes across multiple waves of data collection simultaneously, while also controlling for prior levels of the variables and better accounting for potential error in the modeling. ${ }^{28}$ SEM also allows for assessments of model goodness of fit $^{29}$ and handling of missing data are using full information maximum likelihood estimation.

Based on prior evidence showing different cross-sectional associations between maternal technoference and paternal technoference with child behavior, ${ }^{17}$ we also examined whether model paths and estimates were significantly different for mothers and fathers. Through a multi-group SEM analysis, we first tested whether the statistical fit of the model significantly worsened when we constrained the model paths to be equal across mothers and fathers. If it is found that the fit worsens significantly, this suggests that at least some of the paths in the model must be different for mothers and fathers. We then compared model paths between mothers and fathers to find where they differed from one another and no longer constrained those specific paths to be equal across mothers and fathers.

Finally, in post-hoc analyses, we examined subscales of the $\mathrm{CBCL}$ Internalizing Scale (Emotional Reactivity, Anxiety/Depression, Somatic Complaints, and Withdrawal) and Externalizing Scale (Attention Problems and Aggression) to determine whether specific aspects of child behavior were more strongly associated with parenting stress and parent technoference in our models. As above, these models were tested for differential associations between mothers and fathers.

\section{RESULTS}

Descriptive data are presented in Table 1; for simplicity of interpretation, variables are presented as averages over the four data collection waves. On average, across all four time points, mothers perceived 1.65 devices and fathers perceived 1.43 devices as interfering in their interactions with their child at least once on a typical day; of note, interference was reported as not occurring at all on a daily basis in only $4.9 \%$ of mothers and $9.6 \%$ of fathers. Over half (55.5\%) of mothers and $43.0 \%$ of fathers reported that two or more devices interrupted their parent-child activities on a daily basis. Parents who reported no technoference over any of the time points were very similar to the other parents in our sample in terms of age, ethnicity, marital status, child age, education, and income; only in fathers who reported no technoference at all did we see that these fathers had a higher family income as compared with other fathers $(t(174)=-2.23, p$ $<0.05)$. Maternal and paternal technoference were moderately correlated over the study period $(0.26-0.58$, ps $<0.001)$.
Additionally, maternal reports of technoference were moderately to highly correlated over the six months of the study period $(0.53-0.76, p s<0.001)$, as were paternal reports of technoference over the study period $(0.50-0.84$, $p s<0.001)$. In our sample at baseline, $4 \%$ of parents' ratings of children met or exceeded the clinical cut-off ( $t$-score of 70 or above $)^{21}$ for externalizing behavior and 3\% for internalizing behavior.

Tables 2 and 3 show bivariate associations between parent average technoference, parent/child characteristics, and other study variables. In mothers, younger mother age was associated with higher technoference, and in fathers, greater child media use was associated with greater technoference. Parent race/ethnicity, education level, family income, and child age were not associated with technoference levels (see Table 2). In both mothers and fathers, higher technoference was associated with greater child externalizing and internalizing behaviors, and higher parenting stress; higher technoference was also associated with lower coparenting quality (fathers only) and greater parent depressive symptoms (mothers only) (see Table 3 ).

As described above, we first examined whether the SEM model could be run for all parents (mothers and fathers combined) or whether significant differences emerged in model paths by parent gender utilizing a multi-group analysis. We found that the model fit was significantly worse if all model paths were constrained across parent gender in both the externalizing $\left(\Delta x^{2}(21)=54.14, p\right.$ $<0.001)$ and internalizing models $\left(\Delta x^{2}(21)=53.61, p<0.001\right)$. We therefore examined the pairwise parameter comparisons in AMOS to identify which paths were significantly different by parent gender. These few paths (displayed as mother/father estimates in Figs. 1 and 2) were then allowed to be freely estimated for mothers and for fathers in the model, while all other paths were constrained to be equal across parent gender. The final model for technoference, parent stress, and child externalizing behavior fit the data well $\left(X^{2}(73)=109.53, p<0.01\right.$; $\left.\operatorname{RMSEA}=0.03 ; \mathrm{CFI}=0.99\right)$ as did the model for internalizing behavior $\left(X^{2}(73)=190.60, p<\right.$ 0.001 ; $\mathrm{RMSEA}=0.06 \mathrm{CFI}=0.95$ )

\section{$\mathrm{H1}$ : frequency of technoference would predict greater child} behavior problems

We found support for our hypothesis $(\mathrm{H} 1)$ in the child externalizing behavior model, with technoference in parent-child interactions predicting greater externalizing behavior at each of the following time points $(\beta s=0.11,0.16$, and $0.13, p s<0.01)$. $\mathrm{H} 1$ was only partially supported for child internalizing behavior, with only month three technoference predicting greater month six internalizing $(\beta=.21, p<0.001)$.

\section{$\mathrm{H} 2$ : child behavior problems would predict greater parenting} stress

We found support for our hypothesis $(\mathrm{H} 2)$ in the child externalizing behavior model, with child externalizing predicting greater parenting stress at each of the following time points $(\beta s=0.16$, 0.15 , and 0.12 , ps $<0.01$ ). $\mathrm{H} 2$ was only partially supported for child internalizing behavior, with internalizing at baseline predicting greater parenting stress at month one $(\beta=0.13, p<0.01)$.

H3: greater parenting stress would predict greater technoference We found some support for our hypothesis $(\mathrm{H} 3)$ in both the externalizing and internalizing models, as parenting stress predicted later technoference from baseline to month 1 ( $\beta \mathrm{s}=$ 0.19 and 0.15 , ps $<0.01$ ) and from month one to month three ( $\beta s$ $=0.17$ and 0.19 , ps $<0.001$ ) in both models. The path from month three parenting stress to month six technoference was in the hypothesized direction but not statistically significant $(p<0.10)$.

Post-hoc analyses on internalizing and externalizing subscales All subscale model results, except for the withdrawal model, were very similar to the overall internalizing or externalizing models. To 


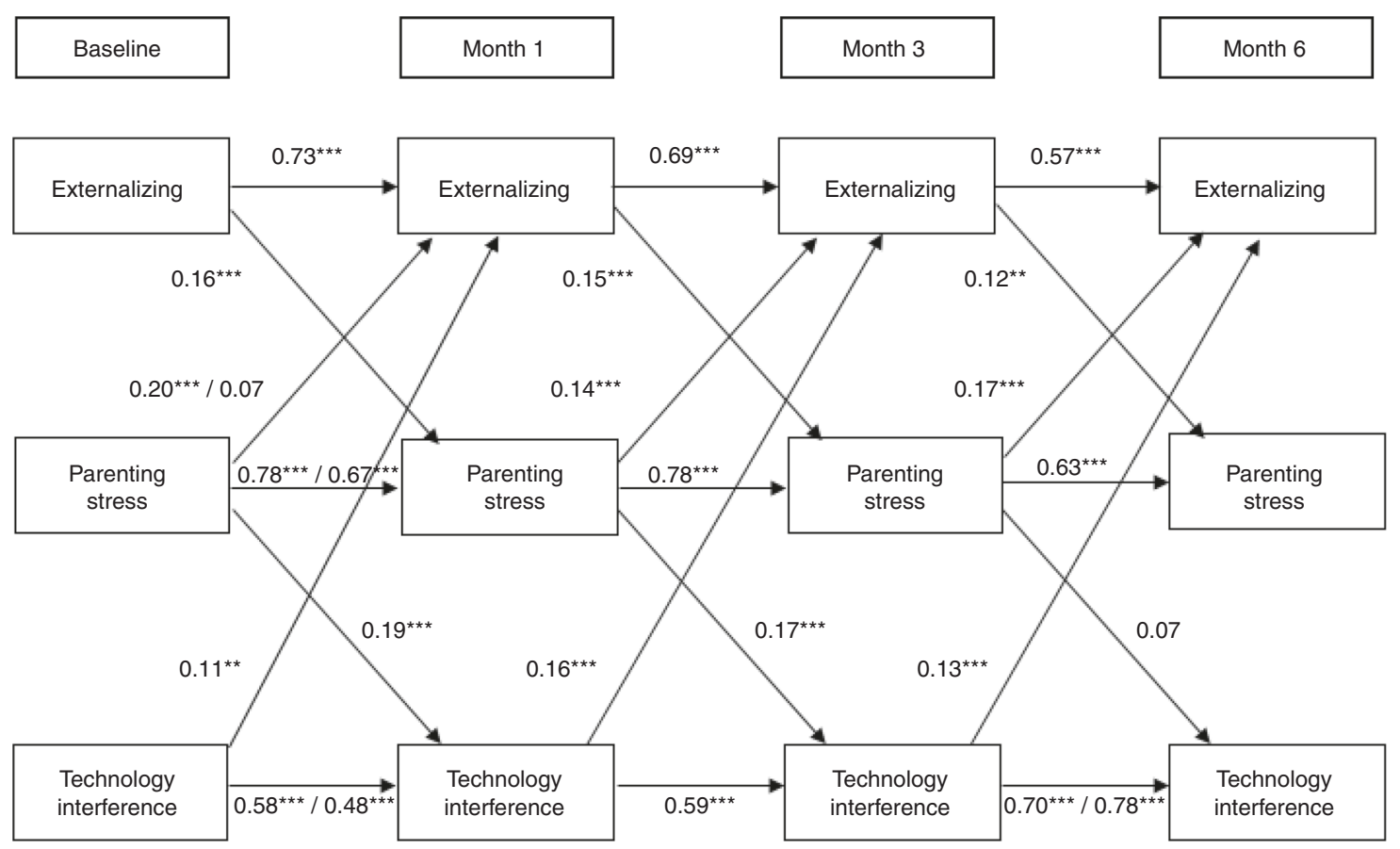

Fig. 1 Structural equation model of longitudinal associations between parent-reported technology interference in the parent-child relationship and child externalizing behavior, with parenting stress as the mediator between externalizing behavior and later parent-child technology interference. Standardized path estimates are displayed. Mothers' and fathers' estimates are displayed as mother/father when found to be significantly different between mothers and fathers; all other model paths were constrained to be equal between mothers and fathers. Parent characteristics, child age, child screen use, parent depressive symptoms, and coparenting quality were controlled but then removed as results did not change. ${ }^{* * *} p<0.001,{ }^{* *} p<0.01,{ }^{*} p<0.05$

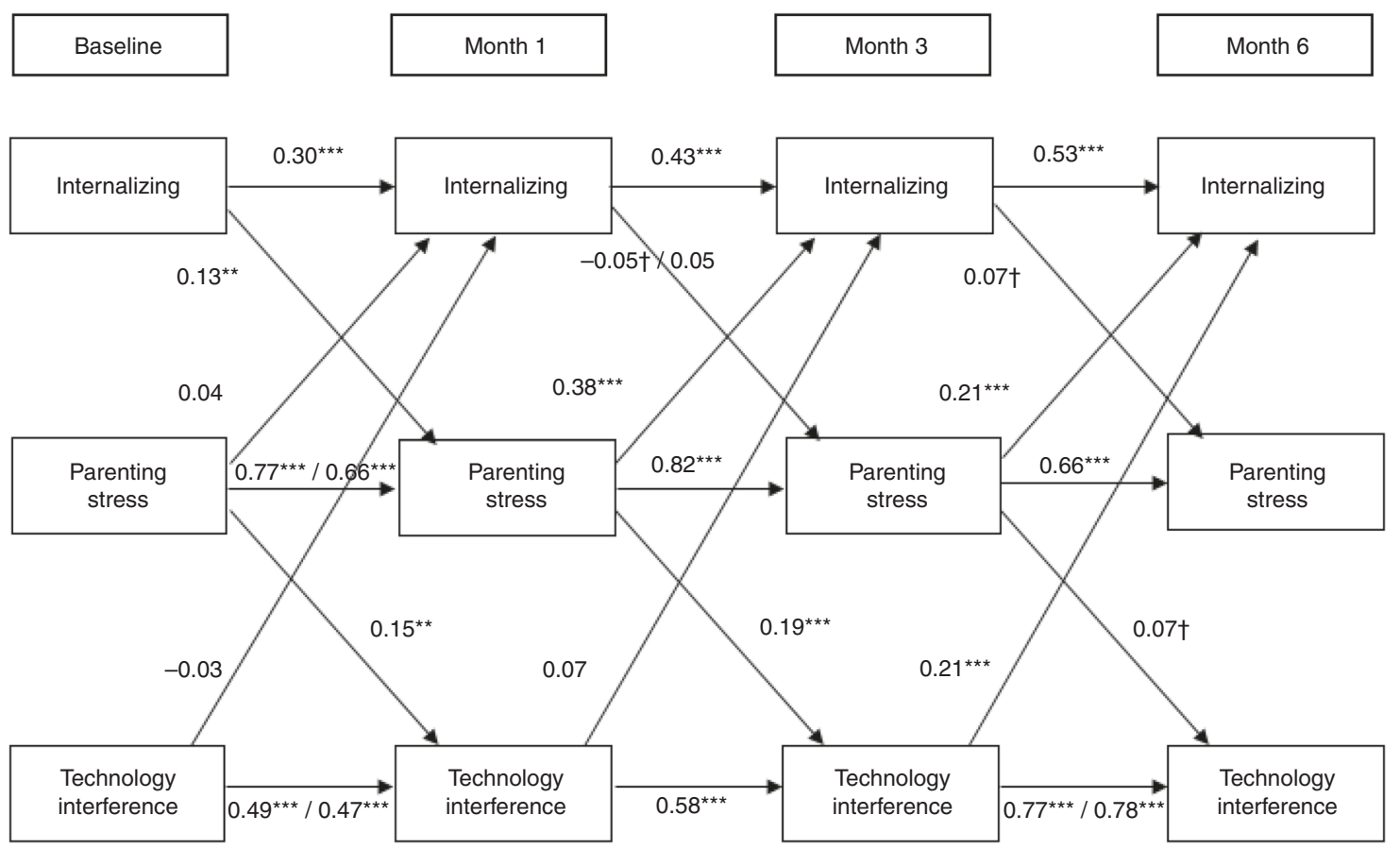

Fig. 2 Structural equation model of longitudinal associations between parent-reported technology interference in the parent-child relationship and child internalizing behavior, with parenting stress as the mediator between internalizing behavior and later parent-child technology interference. Standardized path estimates are displayed. Mothers' and fathers' estimates are displayed as mother/father when found to be significantly different between mothers and fathers; all other model paths were constrained to be equal between mothers and fathers. Parent characteristics, child age, child screen use, parent depressive symptoms, and coparenting quality were controlled but then removed as results did not change. ${ }^{* * *} p<0.001,{ }^{* *} p<0.01,{ }^{*} p<0.05,+p<0.10$ 
Table 1. Descriptive information on parent-reported technoference, child behavior ratings, and other study variables (averaged across all time points)

\begin{tabular}{|c|c|c|c|c|c|c|}
\hline & \multicolumn{2}{|c|}{ Mothers } & \multicolumn{2}{|c|}{ Fathers } & $\begin{array}{l}\text { Correlation between mothers \& } \\
\text { fathers }\end{array}$ & $\begin{array}{l}\text { Diff. between mothers } \& \text { fathers } \\
\text { t-value }\end{array}$ \\
\hline Technoference & 0.44 & $(0.39)$ & 0.38 & $(0.51)$ & $0.51^{* * *}$ & $1.88^{\mathrm{a}}$ \\
\hline $\begin{array}{l}\text { Number of devices that interfere at least once } \\
\text { per day, } n(\%)\end{array}$ & 1.65 & $(0.98)$ & 1.43 & $(1.15)$ & $0.23^{* *}$ & $2.18^{*}$ \\
\hline None & 9 & $4.9 \%$ & 17 & $9.6 \%$ & & \\
\hline Three or more & 32 & $17.6 \%$ & 29 & $16.4 \%$ & & \\
\hline \multicolumn{7}{|l|}{ Child behavior ratings } \\
\hline Externalizing (CBCL; $t$-scored) & 45.22 & $(9.97)$ & 45.19 & $(10.52)$ & $0.65^{* * *}$ & 0.05 \\
\hline Internalizing (CBCL; $t$-scored) & 41.08 & $(11.43)$ & 40.58 & $(11.88)$ & $0.61^{* * *}$ & 0.64 \\
\hline \multicolumn{7}{|l|}{ Other study variables } \\
\hline
\end{tabular}

reduce redundancy, we therefore only report the model results for the withdrawal model here $\left(x^{2}(73)=201.26, p<0.001\right.$; $\mathrm{RMSEA}=$ $0.06 \mathrm{CFI}=0.95)$. These withdrawal model results were also similar to the overall internalizing model; however, a few more model paths were significant (see Fig. 3). In the withdrawal model, we found partial support for our hypothesis $(\mathrm{H} 1)$, with technoference in parent-child interactions predicting greater withdrawal behavior at both month 1 and month 6 ( $\beta s=0.09$ and $0.32, p s<0.01)$. We found partial support for our hypothesis $(\mathrm{H} 2)$, with greater child withdrawal predicting greater parenting stress at both month one and month six ( $\beta s=0.18$ and $0.10, p s<0.05)$. Finally, we found support for our hypothesis $(\mathrm{H} 3)$, as greater parenting stress predicted greater technoference at month one and three ( $\beta s=0.18$ and $0.16, p s<0.001)$ and at a trend level at month six $(\beta$ $=0.07, p<0.10)$.

\section{DISCUSSION}

This study is the first to show longitudinal associations between mother and father technology use during parent-child activities and reported child behavioral difficulties, with evidence of bidirectional associations between technoference and child externalizing symptoms over the course of six months. Although technoference was sometimes associated with greater child internalizing symptoms, bidirectional associations between internalizing symptoms, parenting stress, and technoference were most consistent with regard to child withdrawal.

The bidirectional associations documented in this study are consistent with existing literature. First, our models show evidence for high parent technology use predicting small but significant increases in externalizing child behavioral difficulties, which is consistent with public observations of children behaviorally escalating to get the attention of parents absorbed in their mobile devices. In naturalistic observations, Radesky and colleagues described recurrent instances of young children acting silly, raising their voices, and showing more impulsive behaviors while their caregivers' attention was attuned to a mobile device during fast food meals. ${ }^{14}$ A recent study of families' mobile device use patterns revealed that children are often frustrated by the sudden withdrawal of parental attention when responding to a notification on a mobile device, especially if the reason for device use is unclear. ${ }^{3}$

Another less immediate mechanism is that, when mobile device use displaces verbal and nonverbal interaction ${ }^{15}$ and responsiveness over time, ${ }^{16}$ it is possible that children receive less parent scaffolding-the parent's ability to give the child just enough positive support to perform a new skill on their own-in developing behavior regulation. In order to effectively scaffold child social and emotional skills, parents need to understand the child's mental state and motivations for behavior, in order to intervene effectively to help the child calm down, identify feelings, and problem-solve. ${ }^{30}$ However, parents who frequently use mobile devices during parent-child activities showed lower understanding of their child's mental state and intentions. ${ }^{31}$ Work in pre-adolescents suggests that screen media use displaces faceto-face interactions in such a way as to make it difficult to read others' nonverbal social cues, and when digital media are restricted, children get better at interpreting others' emotional states. ${ }^{32}$ The same mechanism could be occurring with parents and their young children, whose social cues are not always easy or clear to interpret. In prior in-depth interviews, parents have described difficulty dividing their attention between children and the "always-on" work or social demands of their mobile device, making it challenging to read and respond to child behavioral and emotional cues contingently. ${ }^{2}$

Conversely, our models showed that child behavioral difficulties -especially externalizing-were associated with later higher levels of parent stress, which in turn were associated with higher downstream technology use during parent-child activities. Mobile and traditional media have long been considered ways that adults relieve stress, ${ }^{33}$ regulate boredom or anxiety, ${ }^{34}$ or withdraw from social interactions. ${ }^{3,35}$ Mobile communication researchers posit that aspects of interactive design act upon brain reward circuits in such a way that they induce pleasure ${ }^{36}$ and habit-formation. ${ }^{37}$ The same concepts hold for parents; in qualitative interviews, many stay-at-home mothers reported using digital technology as a way to "escape" the boredom or frustrations of childrearing, or to regulate their own emotions or arousal; ${ }^{2}$ reaching out to friends, catching up on the news, or playing games were described as ways to take breaks from sometimes exhausting home routines. 
Table 2. Baseline demographic characteristics and associations with parent-reported technoference (averaged across all time points)

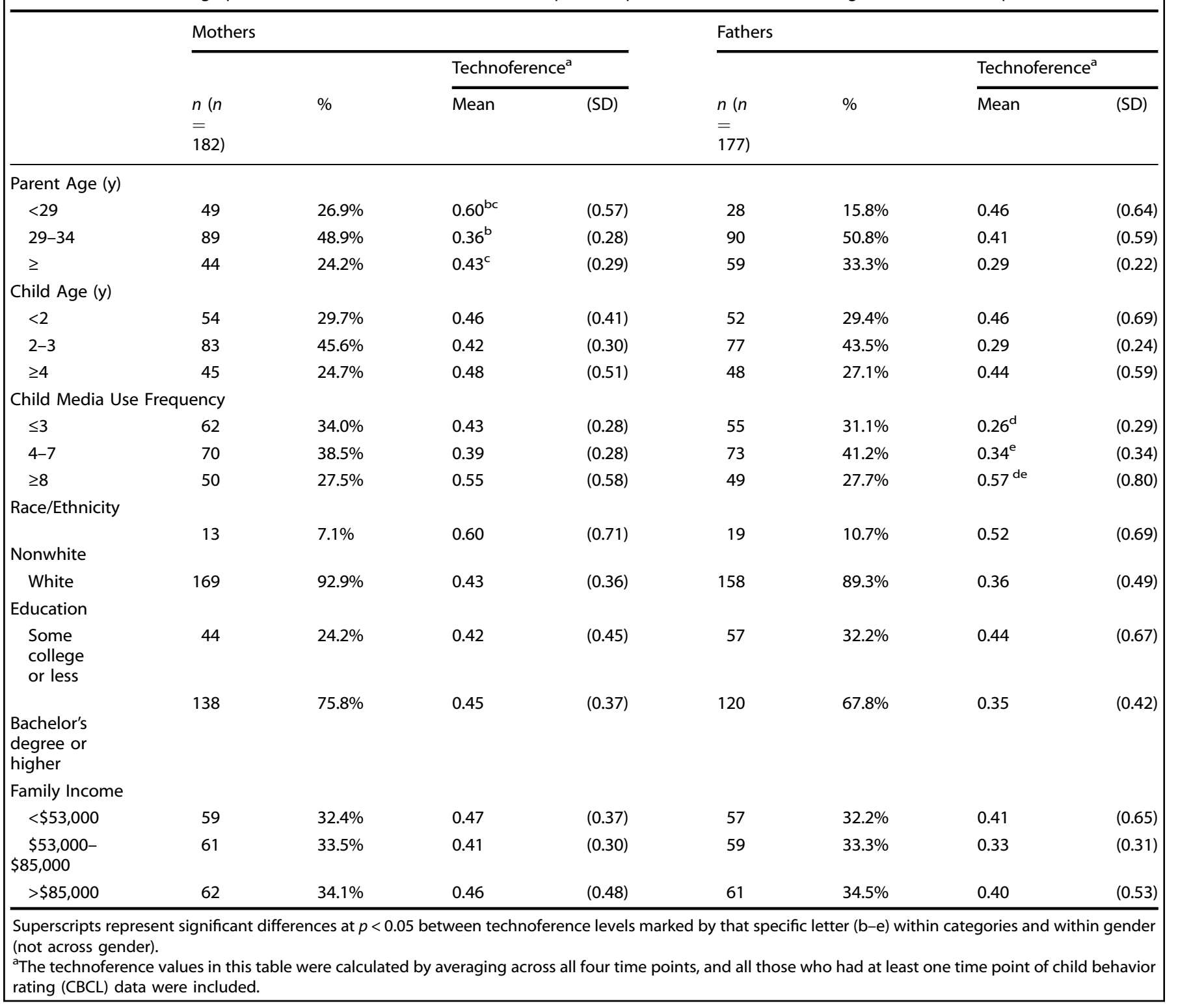

By demonstrating parenting stress as mediator of these associations, our findings support a conceptual model in which parents use digital technology devices as a potential means of escape and for stress management. ${ }^{2} \mathrm{~A}$ recent ecologic examination of everyday mobile device use within the family context revealed that parents sometime use mobile devices as a way to actively withdraw from parenting duties-pretending to be occupied with something important when children need help-and that this constituted a "desirable disengagement" to find time for oneself amid parenting demands. ${ }^{3}$

Overall, like most developmental processes, these results support the hypothesis that relationships between parent technoference and child externalizing behavior are transactional ${ }^{19}$ and influence each other over time. In other words, parents who have children with more externalizing problems become more stressed, which may lead to greater technoference (e.g., withdrawal with technology), which in turn may contribute to more child externalizing problems (and only sometimes internalizing problems). Our results suggest that children may be more likely to act out over time in response to technoference as opposed to internalize, although when we examined internalizing subscales, child withdrawal was the most consistently associated with parent technoference over time. This may be due to (1) parents responding to child withdrawal social cues by feeling they too can disengage into their mobile device use, or (2) parent media use precipitating child withdrawal from social interaction, which was observed in prior observational work. ${ }^{14}$

It is possible that greater parent digital technology use may also be a marker of other parent or household characteristics that independently predict digital technology use, parenting stress, and child behavioral problems, such as greater family dysfunction, ${ }^{38}$ parent mental health or anxiety symptoms, ${ }^{39}$ or work-life balance difficulties ${ }^{40}$-although controlling for parent and child characteristics, as well as parent depression and coparenting quality, did not change our model results significantly. However, we postulate that specific aspects of digital technology, including persuasive design elements (i.e., design aspects that reward the user for prolonged engagement), ${ }^{41}$ are particularly appealing to parents with their own self-regulation difficulties ${ }^{42}$ or those frustrated with their family social environment, ${ }^{2,3}$ which could 
Table 3. Associations between parent-reported technoference and main study variables (averaged across all time points)

\begin{tabular}{|c|c|c|c|c|c|c|c|c|}
\hline & \multicolumn{4}{|l|}{ Mothers } & \multicolumn{4}{|l|}{ Fathers } \\
\hline & $n(n=182)$ & $\%$ & Mean & $(\mathrm{SD})$ & $n(n=177)$ & $\%$ & Mean & $(\mathrm{SD})$ \\
\hline \multicolumn{9}{|c|}{ Externalizing (CBCL; $t$-scored $)^{a}$} \\
\hline Low $(<44)$ & 91 & $50.0 \%$ & $0.37^{\mathrm{b}}$ & $(0.26)$ & 85 & $48.0 \%$ & $0.30^{f}$ & (0.34) \\
\hline High ( $\geq 44)$ & 91 & $50.0 \%$ & $0.52^{\mathrm{b}}$ & $(0.48)$ & 92 & $52.0 \%$ & $0.46^{f}$ & $(0.62)$ \\
\hline Low $(<41)$ & 92 & $50.5 \%$ & $0.37^{c}$ & $(0.24)$ & 89 & $50.3 \%$ & $0.31^{\mathrm{g}}$ & $(0.34)$ \\
\hline High ( $\geq 41)$ & 90 & $49.5 \%$ & $0.52^{c}$ & $(0.49)$ & 88 & $49.7 \%$ & $0.45^{\mathrm{g}}$ & (0.64) \\
\hline \multicolumn{9}{|c|}{ Parenting stress ${ }^{a}$} \\
\hline Low $(<2)$ & 95 & $52.2 \%$ & $0.38^{d}$ & $(0.25)$ & 95 & $53.7 \%$ & $0.30^{\mathrm{h}}$ & $(0.30)$ \\
\hline High $(\geq 2)$ & 87 & $47.8 \%$ & $0.51^{d}$ & $(0.49)$ & 82 & $46.3 \%$ & $0.47^{h}$ & $(0.67)$ \\
\hline \multicolumn{9}{|c|}{ Depressive symptoms ${ }^{a}$} \\
\hline Low $(<5)$ & 79 & $43.4 \%$ & 0.49 & $(0.51)$ & 84 & $47.5 \%$ & $0.49^{i}$ & (0.69) \\
\hline High $(\geq 5)$ & 103 & $56.6 \%$ & 0.41 & $(0.27)$ & 93 & $52.5 \%$ & $0.28^{i}$ & (0.24) \\
\hline
\end{tabular}

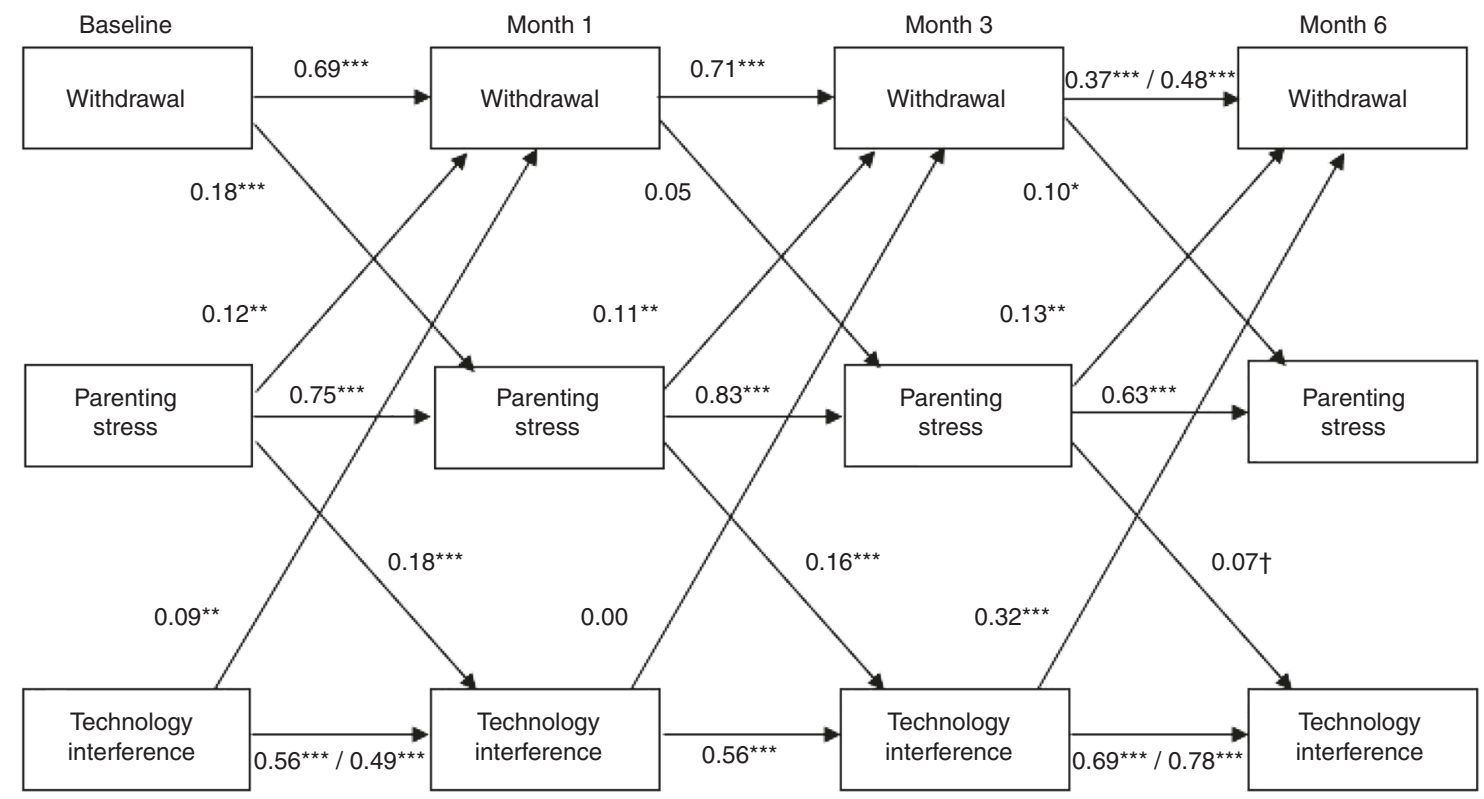

Fig. 3 Structural equation model of longitudinal associations between parent-reported technology interference in the parent-child relationship and child withdrawal behavior, with parenting stress as the mediator between withdrawal behavior and later parent-child technology interference. Standardized path estimates are displayed. Mothers' and fathers' estimates are displayed as mother/father when found to be significantly different between mothers and fathers; all other model paths were constrained to be equal between mothers and fathers. ${ }^{* * *} p<0.001,{ }^{* *} p<0.01,{ }^{*} p<0.05,+p<0.10$

lead to more technology interruptions than would otherwise occur. The technoference method of assessing media use is particularly relevant to this hypothesis, since it characterizes the invasion or interruption of life routines with mobile device use, through notifications made by the phone or ad-hoc habitual usage on the part of the user. However, experimental studies will be necessary to test these hypotheses.
We recognize that the primary limitation of this study was the use of parent self-reports of digital technology use and child behavior, rather than observational methods, which could lead to single-reporter bias. However, self-report methods allow examination of mobile device use by both parents over recurrent time points, which has not been studied in prior publications, $2,14,15$ and allow feasible data collection within a larger sample size. Although 
effects were generally small in size, their consistent associations over time, particularly for child externalizing behavior, suggest that cumulative effects on parent, or child behavior could become clinically relevant. Additionally, it is possible that internalizing behaviors might be more likely than externalizing behaviors to go unnoticed by parents distracted by technology, potentially leading to the differing results in the externalizing and internalizing models. Future observational work will be necessary to objectively characterize children's reactions to parent mobile device use. This study was also limited by having a primarily Caucasian, cohabiting, fairly-educated sample, so results may not be generalizable to the entire U.S. population, but its findings are an important contribution to the understanding of complex family processes around rapidly adopted digital technologies. Our findings also emphasize the importance of technology interference in relatively well-educated families, who are not often conceived as being 'at risk' in terms of high media use. ${ }^{9}$ Given these findings' relevance for understanding contextual influences on child behavior and for crafting clinical recommendations, they should be replicated in larger and more diverse cohorts.

\section{CONCLUSION}

This study's findings have several implications for future research and clinical work. Given the transactional nature of technology use and child development/behavior, future studies should consider using statistical approaches that allow examination of bidirectional associations. It will be important to measure how parents are using mobile devices throughout the day, including content and motivations for use (e.g., entertainment, work-related). Moreover, sequential analysis of parent-child interaction during mobile device use will be helpful to determine whether, moment to moment, child behavioral difficulties appear to precipitate or stem from parent technology use.

Clinically, our results suggest that mobile devices and other digital technology are potentially serving stress-relieving purposes for parents, but at the same time potentially displacing opportunities for parent-child connection important to child health and development. In addition to parents setting their own media limits-for example through the American Academy of Pediatrics' Family Media Use Plan-it may be important to help parents build awareness regarding their relationship with technology and how media influences family dynamics. At the same time, it will also be necessary to provide skills for managing difficult child behavior and reducing parenting stress. However, because our study suggests small but significant long-term associations between technoference and child externalizing and internalizing behavior, it would be worthwhile to study whether experimental manipulation of parent mobile phone use habits-for example through unplugged family routines or less intrusive digital design $^{43}$-might lead to improvements in the parent-child relationship and child behavior.

\section{FUNDING}

College of Health and Human Development, Department of Human Development and Family Studies, and the Bennett Pierce Prevention Research Center at The Pennsylvania State University; NIDA (T32DA017629); NICHD (F31 HD084118).

\section{ADDITIONAL INFORMATION}

Competing interests: The authors declare no competing interests.

Publisher's note: Springer Nature remains neutral with regard to jurisdictional claims in published maps and institutional affiliations.

\section{REFERENCES}

1. Ling R. The mobile connection: the cell phone's impact on society. (Morgan Kaufmann, Massachusetts, 2004).

2. Radesky, J. S. et al. Parent perspectives on their mobile technology use: the excitement and exhaustion of parenting while connected. J. Dev. Behav. Pediatr. 37, 694-701 (2016)

3. Oduor E., Neustaedter C., Odom W., Tang A., Moallem N., Tory M., et al. (eds.) The frustrations and benefits of mobile device usage in the home when co-present with family members. In Proc. of the 2016 ACM Conference on Designing Interactive Systems (ACM, New york, 2016).

4. McDaniel, B. T. \& Coyne, S. M. "Technoference": The interference of technology in couple relationships and implications for women's personal and relational wellbeing. Psychol. Pop. Media Cult. 5, 85 (2016).

5. McDaniel, B. T., Galovan, A. M., Cravens, J. D. \& Drouin, M. "Technoference" and implications for mothers' and fathers' couple and coparenting relationship quality. Comput. Human. Behav. 80, 303-313 (2018).

6. Przybylski, A. K. \& Weinstein, N. Can you connect with me now? How the presence of mobile communication technology influences face-to-face conversation quality. J. Soc. Pers. Relat. 30, 237-246 (2013).

7. Misra, S., Cheng, L., Genevie, J. \& Yuan, M. The iPhone effect: the quality of inperson social interactions in the presence of mobile devices. Environ. Behav. 48, 275-298 (2016).

8. Lauricella A. R., et al. The common sense census: plugged-in parents of tweens and teens (Common Sense Media, California, 2017).

9. Wartella E., Rideout, V., Lauricella, A. Parenting in the Age of Digital Technology http://cmhd.northwestern.edu/wp-content/uploads/2015/06/ParentingAgeDigital Technology.REVISED.FINAL_.2014.pdf. 2014. Accessed date October 1, 2017.

10. McDaniel, B. T. \& Coyne, S. M. Technology interference in the parenting of young children: implications for mothers' perceptions of coparenting. Soc. Sci. J. 53, 435-443 (2016).

11. Bornstein, M. H., Tamis-Lemonda, C. S., Hahn, C. S. \& Haynes, O. M. Maternal responsiveness to young children at three ages: longitudinal analysis of a multidimensional, modular, and specific parenting construct. Dev. Psychol. 44, 867-874 (2008).

12. Johnson, S. B., Riley, A. W., Granger, D. A. \& Riis, J. The science of early life toxic stress for pediatric practice and advocacy. Pediatrics 131, 319-327 (2013).

13. Davidov, M. \& Grusec, J. E. Untangling the links of parental responsiveness to distress and warmth to child outcomes. Child Dev. 77, 44-58 (2006).

14. Radesky, J. S. et al. Patterns of mobile device use by caregivers and children during meals in fast food restaurants. Pediatrics 133, e843-e849 (2014).

15. Radesky, J. et al. Maternal mobile device use during a structured parent-child interaction task. Acad. Pediatr. 15, 238-244 (2015).

16. Hiniker A., et al. (eds) Texting while parenting: how adults use mobile phones while caring for children at the playground. In Proc. of the 33rd Annual ACM Conference on Human Factors in Computing Systems (ACM, New York, 2015).

17. McDaniel, B. T. \& Radesky, J. S. Technoference: parent distraction with technology and associations with child behavior problems. Child Dev. 89, 100-109 (2018).

18. McDaniel, B. T., Coyne, S. M. \& Holmes, E. K. New mothers and media use: associations between blogging, social networking, and maternal well-being. Matern. Child Health J. 16, 1509-1517 (2012).

19. Sameroff, A. Transactional models in early social relations. Hum. Dev. 18, 65-79 (1975).

20. McDaniel B. T. "Technoference":Everyday intrusions and interruptions of technology in couple and family relationships. Family communication in the age of digital and social media (Peter Lang Publishing, New York, 2015).

21. Achenbach T. M., Rescorla L. A. Manual for the ASEBA preschool forms \& profiles: An integrated system of multi-informant assessment; Child behavior checklist for ages 1 1/2-5; Language development survey; Caregiver-teacher report form. (University of Vermont; Burlington, 2000).

22. Abidin, R. R. Parenting stress index. (Psychological Assessment Resources. Inc, Odessa, FL, 1995).

23. Feinberg, M. E., Brown, L. D. \& Kan, M. L. A multi-domain self-report measure of coparenting. Parenting 12, 1-21 (2012).

24. Murphy, S. E., Jacobvitz, D. B. \& Hazen, N. L. What's so bad about competitive coparenting? Family-level predictors of children's externalizing symptoms. J. Child Fam. Stud. 25, 1684-1690 (2016).

25. Radloff, L. S. The CES-D scale a self-report depression scale for research in the general population. Appl. Psychol. Meas. 1, 385-401 (1977).

26. McDaniel, B. T. \& Teti, D. M. Coparenting quality during the first three months after birth: the role of infant sleep quality. J. Fam. Psychol. 26, 886 (2012).

27. Radesky, J. et al. Media and Young Minds. Pediatrics 138, e20162591 (2016).

28. Arbuckle J. L., Wothke W. Amos 4.0 user's guide (SmallWaters Corporation, Chicago, IL, 1999).

29. Buhi, E. R., Goodson, P. \& Neilands, T. B. Structural equation modeling: a primer for health behavior researchers. Am. J. Health Behav. 31, 74-85 (2007). 
30. Rosenblum K. L., Dayton C. J., McDonough S. Communicating feelings: Links between mothers' representations of their infants, parenting, and infant emotional development. (Cambridge University Press, New York, 2006).

31. Radesky, J. S. L. C., Appugliese, D., Miller, A. L., Lumeng, J. C. \& Rosenblum, K. L. Maternal mental representations of the child and mobile phone use during parent-child mealtimes. J. Dev. Behav. Pediatr. 39, 310-317 (2018). In press.

32. Uhls, Y. T. et al. Five days at outdoor education camp without screens improves preteen skills with nonverbal emotion cues. Comput. Human Behav. 39, 387-392 (2014).

33. Hiniker A., Patel S. N., Kohno T., Kientz J. A. (eds). Why would you do that? Predicting the uses and gratifications behind smartphone-usage behaviors. In Proc of the 2016 ACM International Joint Conference on Pervasive and Ubiquitous Computing (ACM, New York, 2016).

34. Bayer, J. B., Dal Cin, S., Campbell, S. W. \& Panek, E. Consciousness and selfregulation in mobile communication. Hum. Commun. Res. 42, 71-97 (2016).

35. Nakamura, T. The action of looking at a mobile phone display as nonverbal behavior/communication: a theoretical perspective. Comput. Human Behav. 43, 68-75 (2015).

36. Brand, M., Young, K. S. \& Laier, C. Prefrontal control and Internet addiction: a theoretical model and review of neuropsychological and neuroimaging findings. Front. Hum. Neurosci. 8, 375 (2014).
37. Bayer, J. B. \& Campbell, S. W. Texting while driving on automatic: considering the frequency-independent side of habit. Comput. Human. Behav. 28, 2083-2090 (2012).

38. Hinkley, T. et al. Early childhood electronic media use as a predictor of poorer wellbeing: a prospective cohort study. JAMA Pediatr. 168, 485-492 (2014).

39. Cheever, N. A., Rosen, L. D., Carrier, L. M. \& Chavez, A. Out of sight is not out of mind: the impact of restricting wireless mobile device use on anxiety levels among low, moderate and high users. Comput. Human. Behav. 37, 290-297 (2014).

40. Chesley, N. Blurring boundaries? Linking technology use, spillover, individual distress, and family satisfaction. J. Marriage Fam. 67, 1237-1248 (2005).

41. Harris T. How Technology is Hijacking Your Mind - from a Magician and Google Design Ethicist. https://journal.thriveglobal.com/how-technology-hijacks-peoplesminds-from-a-magician-and-google-s-design-ethicist-56d62ef5edf3 (2016). Accessed date October 1, 2017.

42. Van Deursen, A. J., Bolle, C. L., Hegner, S. M. \& Kommers, P. A. Modeling habitual and addictive smartphone behavior: The role of smartphone usage types, emotional intelligence, social stress, self-regulation, age, and gender. Comput. Human. Behav. 45, 411-420 (2015)

43. Hiniker A., Hong S. R., Kohno T., Kientz J. A. (ed.) MyTime: designing and evaluating an intervention for smartphone non-use. In Proc. of the $2016 \mathrm{CHI}$ Conference on Human Factors in Computing Systems (ACM, New York, 2016). 